\title{
Pain perception in schizophrenia: influence of neuropeptides, cognitive disorders, and negative symptoms
}

This article was published in the following Dove Press journal:

Neuropsychiatric Disease and Treatment

6 August 2015

Number of times this article has been viewed

\author{
Małgorzata Urban- \\ Kowalczyk' \\ Justyna Pigońska \\ Janusz Śmigielski ${ }^{3}$ \\ 'Department of Affective and \\ Psychotic Disorders, Medical \\ University of Łódź, Łódź, Poland; \\ ${ }^{2}$ Department of Neurology and \\ Movement Disorders, Medical \\ University of Łódź, Łódź, Poland; \\ ${ }^{3}$ Department of Geriatrics, Healthy \\ Ageing Research Centre (HARC), \\ Medical University of Łódź, Łódź, \\ Poland
}

Correspondence: Małgorzata UrbanKowalczyk

Department of Affective and Psychotic Disorders, Medical University of Łódź,

Czechosłowacka 8/10, 92-216 Łódź, Poland

Tel +4842675 737I

Fax +48 426757403

Email malgorzata.urban I@wp.pl
Objectives: The causes and nature of insensitivity to pain in schizophrenia remain unknown. The role of endorphins and the association of cognitive dysfunction and negative symptoms are postulated.

Methods: In this study, 43 patients with schizophrenia, five first-degree relatives, and 34 healthy controls were examined. Participants' plasma concentrations of substance $\mathrm{P}, \beta$-endorphin, and calcitonin gene-related peptide (CGRP) were assessed. In patients, the Trail-Making Test, the Color Reading Interference Test (Stroop test), and the Positive and Negative Syndrome Scale Negative Syndrome subscale (PANSS N) test were performed. We also evaluated pain threshold using nociceptive reflex (RTIII) testing.

Results: The mean $\beta$-endorphin concentration was about $20 \%$ higher in patients than in healthy controls $(P<0.05)$. CGRP concentrations were significantly higher in patients than in controls $(5.34 \mathrm{ng} / \mathrm{mL}$ versus $4.16 \mathrm{ng} / \mathrm{mL} ; P<0.01)$. Subjects treated with antipsychotic polytherapy had higher concentrations of CGRP than did patients treated with second-generation antipsychotic monotherapy (5.92 ng/mL versus $5.02 \mathrm{ng} / \mathrm{mL} ; P<0.05)$. There were no correlations between any biochemical parameters and Trail-Making Test, Stroop test, and PANSS N scores. There were no differences in RTIII among study groups. Strong negative correlation $(P<0.001)$ was found between PANSS N scores and subjective pain threshold on the right lower limb.

Conclusion: The insensitivity to pain in schizophrenia is a complex phenomenon that is probably not related to changes in nociceptive pathways. Increase in $\beta$-endorphin level may be related to this issue, but it is uncertain if such concentration ensures analgesic effect. It is unknown if patients with schizophrenia in fact experience less pain. Cognitive impairment and excess negative symptoms may strongly influence the patient's expression of pain.

Keywords: schizophrenia, endorphin, substance P, calcitonin gene-related peptide, working memory, negative symptoms

\section{Introduction}

Anecdotal case studies have described patients with schizophrenia and various painful medical conditions - eg, perforated bowel, ${ }^{1}$ peritonitis, ${ }^{2}$ injuries, ${ }^{3,4}$ clinically silent heart infarct ${ }^{5}$ - reporting little or no pain. Serious self-injurious behavior like eye enucleation, ${ }^{6}$ self-laceration, ${ }^{7}$ self-amputation of the hand, ${ }^{8}$ and autocastration ${ }^{9}$ have also been described. Most research studies referring to pain insensitivity in schizophrenia were published before 1980. Results were ambiguous and did not give satisfactory explanations for this phenomenon. Various pain parameters, such as threshold, tolerance, and perception, were assessed in these studies using different types of stimuli, including thermal stimuli, ${ }^{10,11}$ mechanical pressure, needle puncture, skin pinch, ${ }^{12-14}$ electrical stimuli, ${ }^{15,16}$ and imagining the situation causing pain. ${ }^{17}$ In only a few studies did the 
authors used neurophysiological methods to obtain a moreobjective evaluation of pain perception in schizophrenia. ${ }^{15,18}$

Diminished pain sensitivity has been reported in patients in the acute phase of psychosis, as well as in stable patients. It is thought that in patients with severe psychotic symptoms, "psychotic analgesia" occurs. ${ }^{19}$ The cause of hypoalgesia in patients with stable mental state is unknown. Neurolepticinduced analgesia, influence of neuropeptides with analgesic properties, lesions in the pain pathway, severe negative symptoms, and cognitive impairment have been suggested. The hypothesis of an increased level of endogenous opioids was proposed but the results of the few studies, however, are contradictory. ${ }^{20,21}$ The role of other transmitters related to pain physiology in the pathogenesis of hypoalgesia is also possible. In our study, we attempted to investigate pathophysiological, biochemical, and cognitive aspects of pain perception in schizophrenia. We hypothesized that $\beta$-endorphin, substance $\mathrm{P}$ (SP) and calcitonin gene-related peptide (CGRP) play a role in altered pain perception in schizophrenia.

$\beta$-endorphin is the best-known endogenous opioid peptide, and it has strong analgesic properties. The peptide inhibits peripheral somatosensory fibers, especially those involved in nociception. ${ }^{22}$ Neurons containing endorphins modulate the regulation of dopaminergic neuronal activity. The impact of excess of $\beta$-endorphin on the pathogenesis of schizophrenia and on altered pain perception in these disorders was suggested. ${ }^{21}$

SP is an undecapeptide, widely distributed in the brain in structures involved in pain mechanisms. It acts as a transmitter in nociceptive pathways. ${ }^{23}$ A few studies evaluating SP concentration did not reveal differences between patients with schizophrenia and controls. ${ }^{24}$

CGRP plays an important role in causing migraine attack. ${ }^{25}$ Intravenous infusion of CGRP produces a migrainelike headache, and CGRP receptor antagonists are able to stop the migraine attack. ${ }^{26}$ Some authors reported that migraine headache are rarer in patients with schizophrenia than in healthy subjects. ${ }^{19,27}$ Moreover, the release of CGRP from the peripheral and central terminals of sensory neurons can be initiated by noxious mechanical stimulation of the skin, electrical stimulation, or noxious heat. The release of CGRP into the spinal cord can facilitate the release of excitatory neurotransmitters in the dorsal horn, promoting synaptic transmission. The release of CGRP following noxious stimulation suggests an important role of this peptide in the transmission of painful stimuli. ${ }^{28}$

The majority of studies reporting hypoalgesia in schizophrenia evaluated the patient's subjective response to painful stimuli. It should be noted that pain has a strong emotional component, and insensitivity to pain in schizophrenia may reflect a deficit in the patient's expression of emotions. In other words, indifference to pain among patients with schizophrenia may not mean actual hypoalgesia. We decided to use nociceptive reflex (RTIII) to evaluate objective nociceptive thresholds in our study. This polysynaptic reflex of spinal origin is largely controlled, like the subjective pain threshold, by supraspinal influences. Studies carried out in healthy subjects have shown that the stimulation threshold at which this reflex is triggered is correlated with the subject's subjective pain threshold. ${ }^{29}$

\section{Materials and methods}

\section{Participants}

The study was conducted in patients with schizophrenia who were being seen in the mental health center of Babiński Memorial Hospital in Łódź, Poland. To be eligible to participate, patients were required to meet the following inclusion criteria: be 18-40 years of age; meet ICD 10 criteria for a diagnosis of schizophrenia; have had psychosis for no longer than 6 years, according to psychiatric examination and using the MINI" (Mini International Neuropsychiatric Interview) is better; ${ }^{30}$ be in a stable mental state and, if hospitalized, scheduled for discharge on the basis of clinical assessment of psychotic symptoms; be taking antipsychotic agents (haloperidol, olanzapine, quetiapine, risperidone, clozapine, amisulpride, aripiprazole) in therapeutic doses. We also enrolled healthy first-degree adult relatives of patients with schizophrenia and a control group of healthy volunteers. The exclusion criteria for all groups were: age $<18$ years and $>40$ years; taking analgesic, antidepressive, or anticonvulsive drugs in last 8 weeks; drug intoxication in the past; use of a psychoactive substance currently or in the past; mental disorders other than schizophrenia; presence of depressive symptoms upon clinical examination and on the Hamilton Depression Rating Scale (score $>7$ ); peripheral neuropathy; limb trauma in the past; a current diagnosis of diabetes or cancer; degenerative diseases of the musculoskeletal system. In all, 43 patients with schizophrenia (aged 19-40 years) were enrolled on study. The female-to-male ratio was 31:12. All patients met the ICD 10 criteria for a diagnosis of schizophrenia, and the duration of psychosis was no longer than 6 years. All patients were in a stable mental state and chronically received antipsychotic agents (haloperidol, olanzapine, quetiapine, risperidone, clozapine, amisulpride, aripiprazole) in therapeutic doses. Five healthy first-degree relatives (three female, two male; aged 18-29 years) of patients with schizophrenia were also included in the study. The control group comprised 34 healthy volunteers (seven male, 27 female; aged 20-40 years). 
All participants gave their consent prior to their inclusion in the study. This research was approved by Ethics Committee of the Medical University of Łódź (Nr RNN/11/08/KE).

\section{Nociceptive reflex}

The nociceptive reflex (RTIII) test was described previously by Willer ${ }^{29}$ and next modified by Guieu et al..$^{15}$ To perform this test we used an electrical stimulator from Medtronic Meden Inmed and an electromyographic recording device. Patients were placed in a supine position on the abdomen so that they could not see their limbs but could obtain adequate muscle relaxation. Electrical stimulation surface electrodes were applied $2 \mathrm{~cm}$ apart to the external retromalleolar gutter, opposite the sural nerve (nervus suralis). Increased current stimuli starting at $1 \mathrm{~mA}$ were applied (frequency of $50 \mathrm{~Hz}$, amplitude $0.2 \mathrm{mV} / \mathrm{D}$, duration of single stimulus $0.1 \mathrm{~ms}$ ). Reflex motor response was recorded by surface electrodes placed on femoral biceps (musculus biceps femoris caput brevis), which were connected to an electromyographic recording device. The nociceptive threshold was defined as the mean minimal stimulation intensity, expressed in milliamperes, at which a reflex motor response was elicited at $100 \% .{ }^{15}$ In the course of the RTIII test, the subjects also had to signal at which moment the electrical stimulus was perceived as painful. The experimental procedure was performed by a senior neurologist who was blinded to whether the subjects were classified as patients, first-degree relatives, or controls. Participants were given an option to take part in this part of experiment.

\section{Biochemical transmitters, cognitive functions, and negative symptoms of schizophrenia}

To measure plasma SP and CGRP concentration by radioimmunoassay, two $4 \mathrm{~mL}$ samples of peripheral blood were collected from all participants and placed into two clot tubes. Samples were then centrifuged. The resulting serum for SP was stored at $-20^{\circ} \mathrm{C}$ until assay. Plasma for CGRP was stored at $-70^{\circ} \mathrm{C}$ until assay. The SP assay was performed using Bachem's Substance P Kit (S-1153; Bachem AG, Bubendorf, Switzerland), and the assay of CGRP was performed using Bachem's Human CGRP Kit (EK-015-02). To measure $\beta$-endorphin plasma concentration, $3 \mathrm{~mL}$ samples of peripheral blood were collected from all participants. The blood samples were drawn into ethylenediaminetetraacetic acid immediately after being drawn. After centrifugation, the plasma samples were stored at $-20^{\circ} \mathrm{C}$ until assay. The assay of $\beta$-endorphin was performed using DRG Diagnostics $\beta$-Endorphin Kit (RIA 3023; DRG International, Inc, Springfield, NJ, USA).
To assess cognitive function in patients with schizophrenia, the Trail-Making Test (TMT) and Color Reading Interference Test (Stroop test) were performed. The Positive and Negative Syndrome Scale Negative Syndrome subscale (PANSS N) test was used to examine the severity of negative symptoms in patients with schizophrenia.

\section{Statistical analysis}

A statistical analysis of the studied characteristics was performed using parametric and nonparametric tests. The analysis of empirical distributions of the parameters was performed using the Shapiro-Wilk test. Use of parametric tests requires, among others, assumption of normality (Student's $t$-test). Mann-Whitney $U$-test was applied if empirical distribution of the studied characteristics was not consistent with normal distribution. Levene's test was applied because variances were not significantly different. Tukey's test was used to compare means with unequal sample sizes. Multivariate statistical analysis parametric tests, $F$-test analysis of variance, and the nonparametric analysis of variance rank test, were used in this study. Analyses were conducted using STATISTICA v 8.0 (StatSoft, Inc., Tulsa, OK, USA).

\section{Results}

Twenty-eight patients with schizophrenia were treated with second-generation antipsychotics (SGAs) in monotherapy, and 15 patients were treated with polytherapy (SGA + first-generation antipsychotic). The mean PANSS $\mathrm{N}$ score in patients was 23.56 (minimum score 16, maximum score 30 ; standard deviation $=4.49$ ). The mean concentration of $\beta$-endorphin was significantly higher in patients than in healthy controls and in first-degree relatives $(27.59 \pm 11.2 \mathrm{pmol} / \mathrm{L}$ versus $22.16 \pm 6.44 \mathrm{pmol} / \mathrm{L}$ and $21.44 \pm 8.24 \mathrm{pmol} / \mathrm{L}$, respectively; $P<0.05$ ) (Figure 1). There were no statistically significant differences in $\beta$-endorphin concentrations between healthy control and relatives.

The mean concentration of CGRP was significantly higher in patients with schizophrenia compared with the control group and relatives $(5.34 \pm 1.77 \mathrm{ng} / \mathrm{mL}$ versus $4.16 \pm 1.52 \mathrm{ng} / \mathrm{mL}$ and $3.29 \pm 1.02 \mathrm{ng} / \mathrm{mL}$, respectively; $P<0.01$ ) (Figure 2). The concentration of CGRP was significantly higher in patients with schizophrenia treated with polytherapy compared with the subjects treated with SGA in monotherapy (mean CGRP concentrations were $5.92 \mathrm{ng} / \mathrm{mL}$ and $5.02 \mathrm{ng} / \mathrm{mL}$, respectively; $P<0.05$ ).

No differences in SP concentration were observed between patients, their relatives, and healthy controls (mean $0.47 \mathrm{ng} / \mathrm{mL}$ versus $0.43 \mathrm{ng} / \mathrm{mL}$ and $0.46 \mathrm{ng} / \mathrm{mL}$, respectively; 


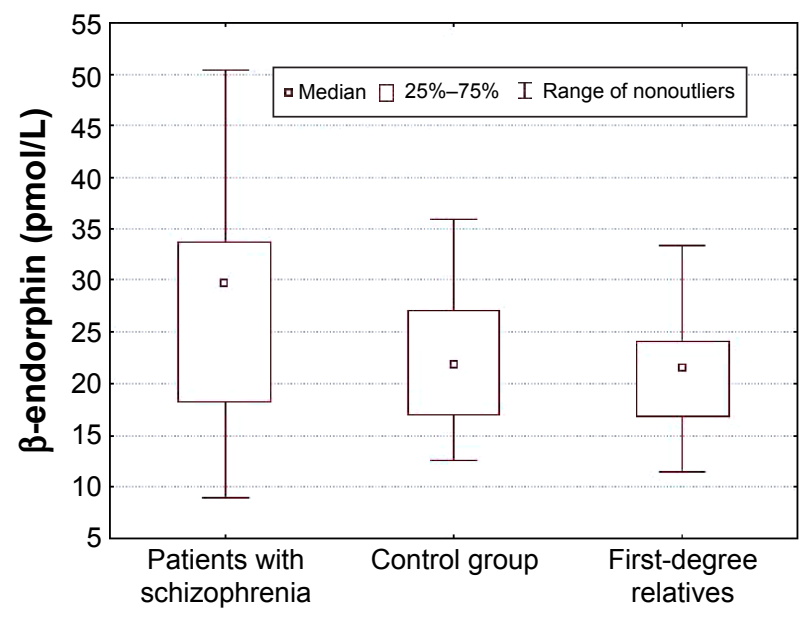

Figure I Comparison of $\beta$-endorphin concentrations in test groups. Notes: Patients with schizophrenia had about 20\% $(P<0.05)$ higher $\beta$-endorphin plasma concentrations than did healthy controls and patients' relatives. Statistical analysis: analysis of variance rank test, Mann-Whitney test.

$P>0.05)$. No correlation was found between the time of performance on the Stroop test and TMT and concentrations of any of the assessed biochemical parameters. In patients, PANSS N scores were not correlated with the concentrations of $\beta$-endorphin, CGRP, or SP.

Nociceptive reflex was evaluated in only 12 patients with schizophrenia, 12 healthy controls, and three first-degree relatives. The remaining participants did not agree to take part in the RTIII test. Study groups did not differ significantly in current intensity at which the stimulus was subjectively perceived as painful or current required to trigger nociceptive reflex. There was no difference in these parameters between the left lower limb and the right lower limb. We

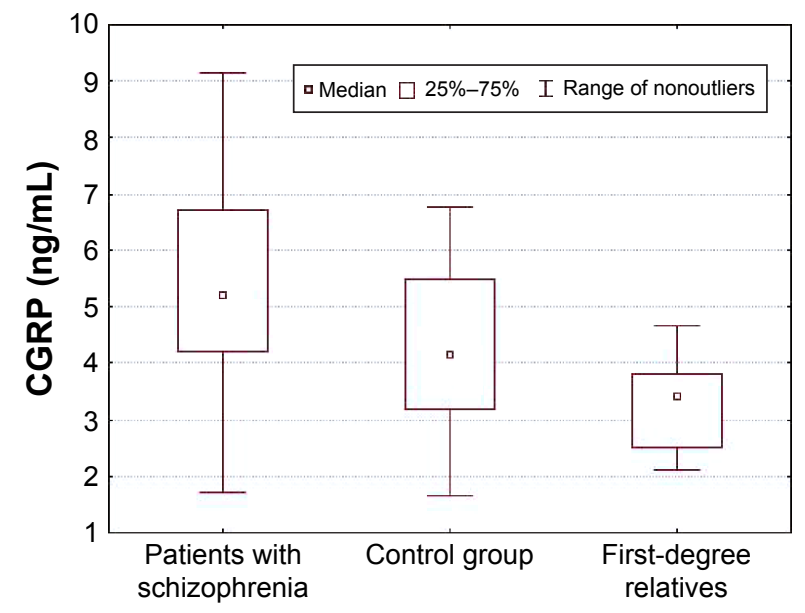

Figure 2 Comparison of CGRP concentrations in test groups.

Notes: Patients with schizophrenia had significantly higher $(P<0.01)$ CGRP serum concentrations than did healthy controls and patients' relatives. Statistical analysis: F-test analysis of variance.

Abbreviation: CGRP, calcitonin gene-related peptide. did not find a statistically significant relationship between the blood $\beta$-endorphin, CGRP, and SP concentrations and currents needed to trigger a painful sensation, and to trigger RTIII in the patients with schizophrenia and in the healthy individuals.

In the patient sample, negative correlation was found between time of performance on the Stroop test and intensity of the current stimulus needed to trigger painful sensation (a subjective pain threshold) in the right lower limb. Additionally, negative correlation was found between time of TMT performance and currents needed to trigger a painful sensation and to trigger RTIII in the right lower limb (Table 1). In other words, those who performed TMT and Stroop test in a shorter time reported a painful sensation at a higher current. Moreover, higher current was necessary to elicit RTIII in these individuals. In patients with schizophrenia, a strong negative correlation $(P<0.001)$ was also found between PANSS $\mathrm{N}$ scores and the intensity of the current needed to trigger a painful sensation on the right lower limb. Negative correlation between PANSS N scores and current intensity needed to trigger RTIII in the right lower limb was shown in this sample (Figures 3 and 4).

\section{Discussion}

The presented study was an attempt to improve the knowledge about hypoalgesia in schizophrenia. Nevertheless, it had methodological limitations. The main problem was the small sample sizes, especially the first-degree relatives group. This sample was too small to draw statistically significant conclusions but, in our opinion, inclusion of this group to the study was valuable for future research. According to our knowledge, in the literature there is only one study in which pain threshold and pain tolerance was examined in relatives of patients with schizophrenia. ${ }^{14}$ In that study, increased pain threshold and increased pain tolerance in relatives was found compared with participants with negative family history of schizophrenia. It should be noted that this study included subjects with varying degrees of kinship, not only first-degree relatives.

Similar to other studies, ${ }^{31,32}$ we showed no significant difference in the concentrations of SP between patients with schizophrenia and healthy subjects. It is probable that SP does not play a critical role in hypoalgesia in schizophrenia. Concentrations of SP in chronic schizophrenia and in neuroleptic-naïve patients with the first episode of psychosis did not differ significantly from concentrations observed in healthy subjects. The results of studies evaluating the level of endogenous opioids are 
Table I The relationship between subjective pain threshold, nociceptive reflex, Stroop test, and TMT results in patients with schizophrenia

\begin{tabular}{|c|c|c|c|c|}
\hline Parameters & $\mathbf{N}$ & $\begin{array}{l}\text { Spearman's rank } \\
\text { correlation }\end{array}$ & Test value & $P$-value \\
\hline LLL pain and Stroop test $\mathrm{tl}$ [s] & 12 & -0.26 & -0.86 & $>0.05$ \\
\hline LLL pain and Stroop test t2 [s] & 12 & -0.60 & -2.34 & $<0.05^{*}$ \\
\hline LLL pain and TMT A t3 [s] & 12 & -0.09 & -0.30 & $>0.05$ \\
\hline LLL pain and TMT B t4 [s] & 12 & -0.22 & -0.70 & $>0.05$ \\
\hline LLL RTIII and Stroop test tI [s] & 12 & -0.00 & -0.01 & $>0.05$ \\
\hline LLL RTIII and Stroop test t2 [s] & 12 & -0.06 & -0.19 & $>0.05$ \\
\hline LLL RTIII and TMT A t3 [s] & 12 & 0.15 & 0.49 & $>0.05$ \\
\hline LLL RTIII and TMT B t4 [s] & 12 & -0.30 & -1.00 & $>0.05$ \\
\hline LLR pain and Stroop test $\mathrm{tl}$ [s] & 12 & -0.78 & -3.96 & $<0.0 I^{*}$ \\
\hline LLR pain and Stroop test t2 [s] & 12 & -0.62 & -2.48 & $<0.05^{*}$ \\
\hline LLR pain and TMT A t3 [s] & 12 & -0.78 & -3.88 & $<0.0 I^{*}$ \\
\hline LLR pain and TMT B t4 [s] & 12 & -0.44 & -1.54 & $>0.05$ \\
\hline LLR RTIII and Stroop test tl [s] & 12 & -0.54 & -2.01 & $>0.05$ \\
\hline LLR RTIII and Stroop test t2 [s] & 12 & -0.37 & -1.26 & $>0.05$ \\
\hline LLR RTIII and TMT A t3 [s] & 12 & -0.61 & -2.42 & $<0.05^{*}$ \\
\hline LLR RTIII and TMT B t4 [s] & 12 & -0.42 & -1.47 & $>0.05$ \\
\hline
\end{tabular}

Note: *Statistically significant.

Abbreviations: LLL pain, left lower limb subjective pain threshold; LLL RTIII, left lower limb nociceptive reflex; LLR pain, lower limb right subjective pain threshold; LLR RTIII, lower limb right nociceptive reflex; [s], time in seconds; tI, Stroop test part I; t2, Stroop test part 2; t3, Trail-Making Test part A; t4, Trail-Making Test part B; TMT, Trail-Making Test.

contradictory, ${ }^{33,34}$ though their role in developing insensitivity to pain in schizophrenia was suggested. ${ }^{19}$ In our study, the mean concentration of $\beta$-endorphin was almost $20 \%$ higher in the patient sample than in the controls. It is difficult to assess whether this concentration is sufficient to ensure significant analgesia.

Patients in our study were in a stable mental state. The comparison of $\beta$-endorphin concentration in acute psychosis and in a state of clinical improvement would be advisable.

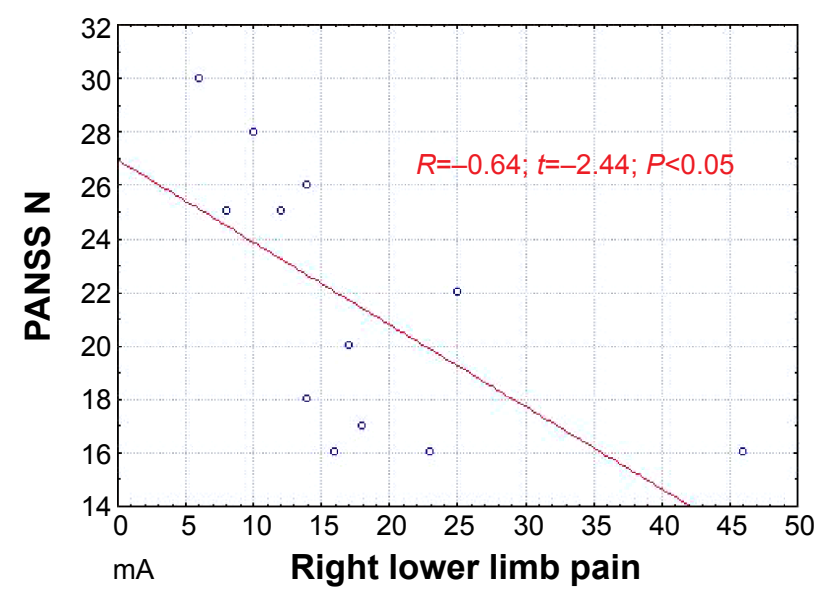

Figure 3 The relationship between PANSS N scores and the intensity of current needed to elicit a painful stimulus in the right lower limb in patients with schizophrenia. Note: Spearman's rank correlation.

Abbreviation: PANSS N, Positive and Negative Syndrome Scale Negative Syndrome subscale.
It should be noted that the increased level of $\beta$-endorphin might be the effect of chronic pharmacotherapy. There is no data on blood opioid level in neuroleptic-naïve populations and in patients with a first episode of psychosis.

According to our knowledge, the presented study is the only one in which the concentration of CGRP was assessed in patients with schizophrenia. In contrast to the expected results, the mean concentration of CGRP was significantly higher in the patient sample compared with the healthy

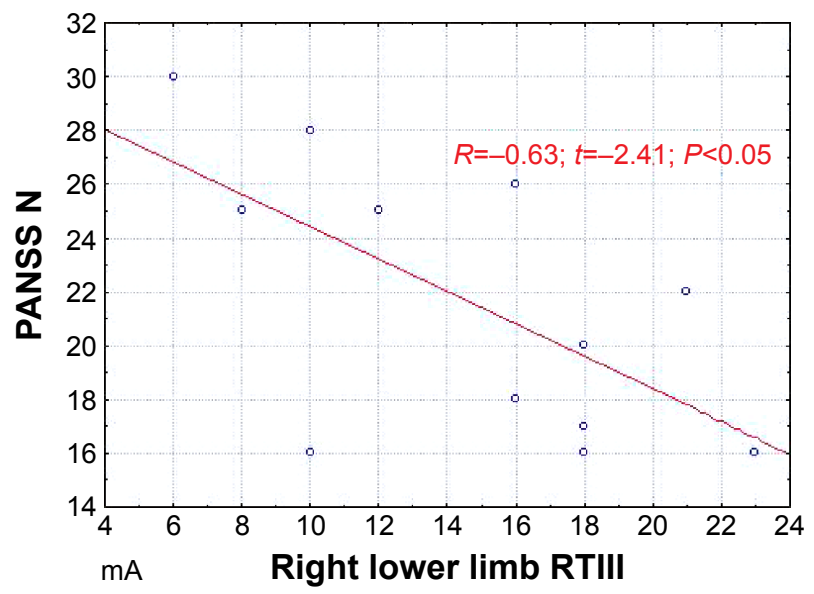

Figure 4 The relationship between PANSS N scores and the intensity of current needed to elicit nociceptive reflex in the right lower limb in patients with schizophrenia. Note: Spearman's rank correlation.

Abbreviations: PANSS N, Positive and Negative Syndrome Scale Negative Syndrome subscale; RTIII, nociceptive reflex. 
controls. We hypothesized that patients would have reduced levels of CGRP, which could at least in part explain the rare occurrence of migraine headaches among this population. Data on the low prevalence of headaches in patients with schizophrenia are derived from single research studies and reports. The results of Kuritzky et $\mathrm{al}^{35}$ do not confirm this information. They estimated the prevalence of headaches and complaints of pain in the group of 108 patients with schizophrenia and 100 healthy individuals. Headaches occurred in $48 \%$ of patients and $41 \%$ of healthy subjects. In addition, up to $40 \%$ of patients experiencing headaches did not complain about them. The authors suggest that patients with schizophrenia tend to have headaches at least as often as healthy subjects but show less willingness to verbalize pain complaints. This is possibly due to the reduced level of emotional awareness of pain, as opposed to the undisturbed ability to identify and locate it. Similar results were also obtained by other researchers. ${ }^{36}$

Some epidemiological, genetic, and clinical data support the hypothesis on the role of autoimmune processes in the pathogenesis of schizophrenia. In patients, deviations and various irregularities in the functioning of the immune system were observed. ${ }^{37}$ It can be assumed that the disorder of the immune system in schizophrenia resembles the changes observed in autoimmune diseases. It is likely that higher concentrations of CGRP in patients in our study were associated with this phenomenon. The role of CGRP was described in numerous conditions associated with chronic inflammation. ${ }^{38,39}$ One possible explanation for the increased levels of CGRP in patients with schizophrenia may be its role in nonspecific stimulation of the immune system described in this disease.

A significant proportion of reported cases of hypoalgesia refer to schizophrenia patients in an acute episode of psychosis, which is associated with increased dopaminergic activity in certain brain structures, including the striatum. Some data suggest analgesic action of dopamine release in this region of the brain. Studies using positron emission tomography showed that the reduced density of striatal D2 receptors in healthy subjects was associated with an increase in pain threshold. ${ }^{40}$ This phenomenon might explain the reduced sensitivity to painful stimuli only in a state of acute psychosis ("psychotic analgesia"). CGRP is involved in dopaminergic transmission in brain. When given intracerebroventricularly, CGRP affects dopamine-related behaviors and potentiates haloperidol-induced catalepsy. ${ }^{42}$ This may suggest the role of CGRP in pathogenesis of schizophrenia. The data indicating that dopamine agonists such as amphetamine and phencyclidine may increase the CGRP microdialysate levels in rat limbic forebrain ${ }^{42}$ support this hypothesis. Angelucci et $\mathrm{al}^{43}$ administered haloperidol, risperidone, and olanzapine to rats and analyzed the concentrations of CGRP in their brains. Haloperidol and higher doses of risperidone decreased the concentration of CGRP in striatum. The antipsychotic agents did not affect the concentration of CGRP in hippocampus, frontal cortex, and hypothalamus. The rats that received olanzapine showed significantly higher concentrations of CGRP in striatum, frontal cortex, and hypothalamus. As in the case of haloperidol and risperidone, there was no effect on peptide levels in the hippocampus. It is likely that the different effects of the studied antipsychotic agents on the level of CGRP result from different receptor mechanisms, especially with the low affinity of olanzapine to D2 receptors in the striatum. These findings may indicate that the antipsychotic action of drugs related to dopaminergic transmission could be partly mediated by a diminished synthesis of CGRP.

In our study, patients treated with polytherapy showed statistically significant higher mean plasma CGRP concentrations compared with those treated only with SGA, which could be associated with a variety of drug receptor mechanisms. These results should be compared with the concentrations of CGRP in patients treated only with a firstgeneration antipsychotic. It is also important that in our study, the most-frequently used combination of antipsychotics in polytherapy was haloperidol and olanzapine. Olanzapine is the only drug so far known to affect the concentration of CGRP. There is no such data for other SGAs, which in this case were comparatively used as often as olanzapine in monotherapy. The role of CGRP in schizophrenia with hypoalgesia remains unclear; however, further research could provide interesting findings.

Our results of RTIII examination are similar to the results obtained by Guieu et $\mathrm{al}^{15}$ who triggered this reflex in ten patients with schizophrenia and ten healthy controls and showed no differences in nociceptive reflex threshold in patients with schizophrenia compared with healthy subjects. In contrast to our study, the patients did not take neuroleptics. We did not use electrical stimulation parameters identical to the parameters chosen by Guieu et al. ${ }^{15}$ More-advanced electrical stimulators make it impossible to program the parameters indicated therein. This makes the test safer and less unpleasant for the participants.

In one published study, ${ }^{14}$ healthy relatives of patients with schizophrenia showed a significantly elevated pain threshold and increased pain tolerance compared with participants with a negative family history of schizophrenia. For this reason, we decided that the inclusion of even a small group of 
relatives might be interesting. We did not observe increased RTIII threshold in this sample. It may indicate that insensitivity to pain in schizophrenia is not the result of alterations of the pain pathway. Potvin et $\mathrm{al}^{44}$ reported that individuals with schizophrenia remain less reactive to prolonged noxious insult despite unaffected pain threshold. They found that patients showed very little pain sensitization in response to repeat or continuous administration of pain stimulus. In contrast, controls demonstrated strong pain sensitization. These observations may indicate that the excitatory spinal or supraspinal pain profiles are affected in schizophrenia. Nevertheless, these findings were based solely on subjective patient reports. Lévesque et $\mathrm{al}^{18}$ evaluated the contribution of spinal and supraspinal processes underlying the lack of pain sensitization described in schizophrenia using RTIII.

Finally, eleven control subjects and 12 patients with paranoid schizophrenia or schizoaffective disorder under neuroleptic treatment were analyzed. No significant difference in withdrawal reflex threshold between study groups was found. Contrary to our study, the authors reported much lower pain threshold in patients than in controls and moreover, patients with schizophrenia showed less subjective pain sensitization than did controls. Additionally, a negative relationship between pain threshold and positive symptoms was described. The authors concluded that decrease in pain sensitization in patients does not depend on disease-induced alterations at the spinal level and may be an intrinsic feature of schizophrenia. This poor sensitivity to prolonged pain coexisting with strong enhance of acute pain may represent the unique, specific response profile with probable supraspinal etiology.

The heterogeneity of methodology in the current study, including the samples and treatment diversity, confound the explanation of the phenomenon of hypoalgesia. The point is whether patients with schizophrenia who reported little or no pain in fact experienced less pain. Pain has a strong emotional component and so it is likely that factors like cognitive impairment - especially working memory and attention disorders, alterations to the recognition of emotions, and negative symptoms - play at least an important role in experiencing pain in schizophrenia. It was reported that those with schizophrenia have a deficit in the identification and categorization of pain, both in themselves and in others. ${ }^{45}$

The impairment of the processing of motivational affective pain aspects may be related to dysfunction of the prefrontal cortex in schizophrenia. ${ }^{46}$ According to this hypothesis, patients as well as healthy individuals may experience pain without behavioral and affective reactions. Guieu et $\mathrm{al}^{15}$ suggested that "indifference" is a more appropriate term than "insensitivity" to describe pain sensitivity disorders in schizophrenia. Dworkin et $\mathrm{al}^{47}$ demonstrated the relationship between affective flattening and pain insensitivity, but other studies did not confirm these results. ${ }^{16}$ Subjects with schizophrenia could have difficulties with differentiation and the emotional experience of noxious (painful), neutral (tactile), or even pleasant stimuli.

The results of our study support this hypothesis. We demonstrated that patients with severe negative symptoms and working memory disturbances described a stimulus as painful even at very low current electrical stimulation. It should be noted that before the test, patients were instructed that the stimulus would be felt initially as tactile, and only later as painful. This relationship was found only for the right limb. This observation could be connected with lateralization disturbances described in schizophrenia ${ }^{48}$ and lateralization of pain perception. It was thought that subjects with schizophrenia are more frequently left-handed than healthy population. ${ }^{49}$ Now it is considered that they are not left-handed, but rather are not right-handed - they show no clear hand preference (mixed type). ${ }^{50-52}$ Right-hemisphere lateralization is postulated in pain sensation, especially the right lateralization of the amygdala, which is a neuronal pain perception modulator. ${ }^{53,54}$ Lateralization of pain sensation is independent of hand preference. ${ }^{55}$ In participants of our study, the lateralization was not determined but it should be considered in future research.

The majority of communications about hypoalgesia in schizophrenia refer to common pain-related situations in clinical practice. On the other hand, scientific analyses of hypoalgesia in schizophrenia, including ours, are usually based on experimentally induced pain and give ambiguous results. Engels et $\mathrm{al}^{56}$ reviewed 14 studies that evaluated clinical pain in schizophrenia. They found that subjects with schizophrenia have a lower prevalence of pain complaints and lower pain intensity compared with patients with other psychiatric disorders and with controls without psychiatric disorders. The comparison of patient response and noxious stimulation in clinical and experimental circumstances may contribute to more-consistent conclusions.

Altered sensitivity to pain in schizophrenia is an interesting and clinically important phenomenon because it may delay diagnosis and treatment of serious, life-threatening medical conditions. It is also interesting in the context of psychopathology, treatment, and a course of schizophrenia. The results of our study did not provide an explanation for pain insensitivity in schizophrenia. Nevertheless, they suggest 
that it is not related to changes in the nociceptive pathways. Future research should take into account neurophysiological, biochemical, and psychopathological aspects of this phenomenon.

\section{Acknowledgments}

Supported by the grant 502-11-849 from Medical University of Łódź, Łódź, Poland and by the grant N N402 269536 from Ministry of Science and Higher Education.

\section{Disclosure}

The authors report no conflicts of interest in this work.

\section{References}

1. Murakami H, Tamasawa N, Yamashita M, et al. Altered pain perception in schizophrenia. Lancet. 2010;375(9717):864.

2. Rosenthal SH, Porter KA, Coffey B. Pain insensitivity in schizophrenia. Case report and review of the literature. Gen Hosp Psychiatry. 1990;12(5):319-322.

3. Murthy BV, Narayan B, Nayagam S. Reduced perception of pain in schizophrenia: its relevance to the clinical diagnosis of compartment syndrome. Injury. 2004;35(11):1192-1193.

4. Virit O, Savas HA, Altindag A. Lack of pain in schizophrenia: a patient whose arm was burned and amputated. Gen Hosp Psychiatry. 2008;30(4):384-385.

5. Marchand WE. Occurrence of painless myocardial infarction in psychotic patients. $N$ Engl J Med. 1955;253(2):51-55.

6. Feldman MD. The challenge of self-mutilation: a review. Compr Psychiatry. 1988;29(3):252-269.

7. Shore D, Anderson DJ, Cutler NR. Prediction of self-mutilation in hospitalized schizophrenics. Am J Psychiatry. 1978;135(11):1406-1407.

8. Schweitzer I. Genital self-amputation and the Klingsor syndrome. Aust N Z J Psychiatry. 1990;24(4):566-569.

9. Myers WC, Nguyen M. Autocastration as a presenting sign of incipient schizophrenia. Psychiatr Serv. 2001;52(5):685-686.

10. Jochum T, Letzsch A, Greiner W, Wagner G, Sauer H, Bär KJ. Influence of antipsychotic medication on pain perception in schizophrenia. Psychiatry Res. 2006;142(2-3):151-156.

11. Kane EM, Nutter RW, Weckowicz TE. Response to cutaneous pain in mental hospital patients. J Abnorm Psychol. 1971;77(1):52-60.

12. Stengel E, Oldham AJ, Ehrenberg AS. Reactions to pain in various abnormal mental states. $J$ Ment Sci. 1955;101(422):52-69.

13. Merskey H, Gillis A, Marszalek KS. A clinical investigation of reactions to pain. J Ment Sci. 1962;108:347-355.

14. Hooley JM, Delgado ML. Pain insensitivity in the relatives of schizophrenia patients. Schizophr Res. 2001;47(2-3):265-273.

15. Guieu R, Samuélian JC, Coulouvrat H. Objective evaluation of pain perception in patients with schizophrenia. Br J Psychiatry. 1994; 164(2):253-255.

16. Blumensohn R, Ringler D, Eli I. Pain perception in patients with schizophrenia. J Nerv Ment Dis. 2002;190(7):481-483.

17. Petrovich DV. Pain apperception in chronic schizophrenics. J Proj Tech. 1960;24:21-27.

18. Lévesque M, Potvin S, Marchand S, et al. Pain perception in schizophrenia: evidence of a specific pain response profile. Pain Med. 2012; 13(12):1571-1579.

19. Dworkin RH. Pain insensitivity in schizophrenia: a neglected phenomenon and some implications. Schizophr Bull. 1994;20(2):235-248.

20. Meuser KT, Dysken MW. Narcotic antagonists in schizophrenia: a methodological review. Schizophr Bull. 1983;9(2):213-225.

21. Volavka J, Davis LG, Ehrlich YH. Endorphins, dopamine, and schizophrenia. Schizophr Bull. 1979;5(2):227-239.
22. Hartwig AC. Peripheral beta-endorphin and pain modulation. Anesth Prog. 1991;38(3):75-78.

23. Chahl LA. Tachykinins and neuropsychiatric disorders. Curr Drug Targets. 2006;7(8):993-1003.

24. De Wied D, Sigling HO. Neuropeptides involved in the pathophysiology of schizophrenia and major depression. Neurotox Res. 2002; 4(5-6):453-468.

25. Benemei S, Nicoletti P, Capone JA, Geppetti P. Pain pharmacology in migraine: focus on CGRP and CGRP receptors. Neurol Sci. 2007; 28 Suppl 2:S89-S93.

26. Lassen LH, Haderslev PA, Jacobsen VB, Iversen HK, Sperling B, Olesen J. CGRP may play a causative role in migraine. Cephalalgia. 2002;22(1):54-61.

27. Mehta D, Wooden H, Mehta S. Migraine and schizophrenia. Am J Psychiatry. 1980;137(9):1126.

28. Bullock CM, Kelly S. Calcitonin gene-related peptide receptor antagonists: beyond migraine pain - a possible analgesic strategy for osteoarthritis? Curr Pain Headache Rep. 2013;17(11):375.

29. Willer JC. Comparative study of perceived pain and nociceptive flexion reflex in man. Pain. 1977;3(1):69-80.

30. Sheehan DV, Lecrubier Y, Sheehan KH, et al. The Mini-International Neuropsychiatric Interview (M.I.N.I.): the development and validation of a structured diagnostic psychiatric interview for DSM-IV and ICD-10. J Clin Psychiatry. 1998;59 Suppl 20:22-33;quiz 34.

31. Heikkilä L, Rimón R, Terenius L. Dynorphin A and substance $P$ in the cerebrospinal fluid of schizophrenic patients. Psychiatry Res. 1990; 34(3):229-236

32. Miller C, Kirchmair R, Troger J, et al. CSF of neuroleptic-naive firstepisode schizophrenic patients: levels of biogenic amines, substance $P$, and peptides derived from chromogranin A (GE-25) and secretogranin II (secretoneurin). Biol Psychiatry. 1996;39(11):911-918.

33. Brambilla F, Facchinetti F, Petraglia F, Vanzulli L, Genazzani AR. Secretion pattern of endogenous opioids in chronic schizophrenia. Am J Psychiatry. 1984;141(10):1183-1189.

34. Pickar D, Vartanian F, Bunney WE, et al. Short-term naloxone administration in schizophrenic and manic patients. A World Health Organization Collaborative Study. Arch Gen Psychiatry. 1982;39(3):313-319.

35. Kuritzky A, Mazeh D, Levi A. Headache in schizophrenic patients: a controlled study. Cephalalgia. 1999;19(8):725-727.

36. Almeida JG, Kurita GP, Braga PE, Pimenta CA. [Chronic pain in schizophrenic patients: prevalence and characteristics]. Cad Saude Publica. 2010;26(3):591-602. Portuguese.

37. Jones AL, Mowry BJ, Pender MP, Greer JM. Immune dysregulation and self-reactivity in schizophrenia: do some cases of schizophrenia have an autoimmune basis? Immunol Cell Biol. 2005;83(1):9-17.

38. Springer J, Geppetti P, Fischer A, Groneberg DA. Calcitonin generelated peptide as inflammatory mediator. Pulm Pharmacol Ther. 2003; 16(3):121-130.

39. Sacchetti M, Micera A, Lambiase A, et al. Tear levels of neuropeptides increase after specific allergen challenge in allergic conjunctivitis. Mol Vis. 2011;17:47-52.

40. Hagelberg N, Jääskeläinen SK, Martikainen IK, et al. Striatal dopamine D2 receptors in modulation of pain in humans: a review. Eur J Pharmacol. 2004;500(1-3):187-192.

41. Clementi G, Grassi M, Valerio C, Prato A, Fiore CE, Drago F. Effects of calcitonin gene-related peptide on extrapyramidal motor system. Pharmacol Biochem Behav. 1992;42(3):545-548.

42. Mathé AA, Hertel P, Nomikos GG, Gruber S, Mathé JM, Svensson TH. The psychotomimetic drugs D-amphetamine and phencyclidine release calcitonin gene-related peptide in the limbic forebrain of the rat. $J \mathrm{Neu}$ rosci Res. 1996;46(3):316-323.

43. Angelucci F, Gruber SH, Caltagirone C, Mathé AA. Differential effects of olanzapine, haloperidol and risperidone on calcitonin gene-related peptide in the rat brain. Neuropeptides. 2008;42(5-6):535-541.

44. Potvin S, Stip E, Tempier A, et al. Pain perception in schizophrenia: no changes in diffuse noxious inhibitory controls (DNIC) but a lack of pain sensitization. J Psychiatr Res. 2008;42(12):1010-1016. 
45. Wojakiewicz A, Januel D, Braha S, Prkachin K, Danziger N, Bouhassira D. Alteration of pain recognition in schizophrenia. Eur J Pain. 2013;17(9):1385-1392.

46. Maeoka H, Matsuo A, Hiyamizu M, Morioka S, Ando H. Influence of transcranial direct current stimulation of the dorsolateral prefrontal cortex on pain related emotions: a study using electroencephalographic power spectrum analysis. Neurosci Lett. 2012;512(1):12-16.

47. Dworkin RH, Clark WC, Lipsitz JD, et al. Affective deficits and pain insensitivity in schizophrenia. Motiv Emot. 1993;17(3):245-276.

48. Crow TJ. Is schizophrenia the price that Homo sapiens pays for language? Schizophr Res. 1997;28(2-3):127-141.

49. Satz P, Green MF. Atypical handedness in schizophrenia: some methodological and theoretical issues. Schizophr Bull. 1999;25(1):63-78.

50. Cannon M, Byrne M, Cassidy B, et al. Prevalence and correlates of mixedhandedness in schizophrenia. Psychiatry Res. 1995;59(1-2):119-125.
51. Deep-Soboslay A, Hyde TM, Callicott JP, et al. Handedness, heritability, neurocognition and brain asymmetry in schizophrenia. Brain. 2010; 133(10):3113-3122.

52. Dragovic M, Hammond G. Handedness in schizophrenia: a quantitative review of evidence. Acta Psychiatr Scand. 2005;111(6):410-419.

53. Ji G, Neugebauer V. Hemispheric lateralization of pain processing by amygdala neurons. J Neurophysiol. 2009;102(4):2253-2264.

54. Carrasquillo Y, Gereau RW. Hemispheric lateralization of a molecular signal for pain modulation in the amygdala. Mol Pain. 2008;4:24.

55. Lugo M, Istúriz G, Lara C, García N, Eblen-Zaijur A. Sensory lateralization in pain subjective perception for noxious heat stimulus. Somatosens Mot Res. 2002;19(3):207-212.

56. Engels G, Francke AL, van Meijel B, et al. Clinical pain in schizophrenia: a systematic review. J Pain. 2014;15(5):457-467.
Neuropsychiatric Disease and Treatment

\section{Publish your work in this journal}

Neuropsychiatric Disease and Treatment is an international, peerreviewed journal of clinical therapeutics and pharmacology focusing on concise rapid reporting of clinical or pre-clinical studies on a range of neuropsychiatric and neurological disorders. This journal is indexed on PubMed Central, the 'PsycINFO' database and CAS,

\section{Dovepress}

and is the official journal of The International Neuropsychiatric Association (INA). The manuscript management system is completely online and includes a very quick and fair peer-review system, which is all easy to use. Visit http://www.dovepress.com/testimonials.php to read real quotes from published authors.

Submit your manuscript here: http://www.dovepress.com/neuropsychiatric-disease-and-treatment-journal 